\title{
Rigidity transitions and constraint counting in amorphous networks: beyond the mean-field approach
}

\author{
Matthieu Micoulaut \\ Laboratoire de Physique Théorique des Liquides, Université Pierre et Marie Curie, Boite 121 \\ 4, Place Jussieu, 75252 Paris Cedex 05, France
}

(October 26, 2018)

\begin{abstract}
We study rigidity transitions in covalent amorphous networks using sizeincreasing cluster approximations and constraint counting algorithms. Possible consequences of the presence of self-organization are examined. The analysis reveals two transitions instead of the usual (mean-field) single transition. One from a floppy to an isostatic rigid phase at a mean coordination number $\bar{r}_{c 1}$ where the number of floppy modes vanishes and a second one from an isostatic to a stressed rigid phase at $\bar{r}_{c 2}$. The value of the two critical mean coordination numbers as well as the width $\Delta \bar{r}=\bar{r}_{c 2}-\bar{r}_{c 1}$ of the intermediate phase depend very strongly on the presence of medium range order elements such as rings.

Pacs: $61.43 . F s-46.30 . \mathrm{Cn}$
\end{abstract}

The notion of constraints and their application to classical macroscopic physics problems such as the stability of bridges and trusses have been introduced and first considered by J.L. Lagrange and J.C. Maxwell [1,2]. On this basis, J.C. Phillips asserted [3,4] some twenty years ago that covalent networks can be mechanically constrained by interatomic valence forces such as bond- stretching and bond-bending and optimal glass formation is attained when the netwok sits at a mechanically critical point. This happens when the constraints $n_{c}$ per atom estimated by Maxwell counting equal the degrees of freedom per atom in 3D, i.e. $n_{c}=3$.

Such mechanical systems have been examined in terms of percolation theory by M.F. Thorpe [5] who showed by a normal mode analysis that the number of zero frequency solutions (floppy modes) $f$ of the dynamical matrix equals $f=3-n_{c}$ and vanishes when the mean coordination number $\bar{r}$ of the network reaches the critical value $\bar{r}_{c}=2.4$. In this meanfield approach, one considers a network of $\mathrm{N}$ atoms composed of $n_{r}$ atoms that are $r$-fold coordinated. The enumeration of mechanical constraints in this system gives $r / 2$ bondstretching constraints and $(2 r-3)$ bond-bending constraints for a $r$-fold coordinated atom. Since then, a certain number of structural possibilities have been taken into account such as rings, broken bond-bending constraints [6] or the effects of one-fold coordinated atoms [7]. 
These powerful ideas have led to the prediction of a floppy to rigid transition in random networks and various examples where rigidity percolation threshold occurs have been reported [8]. Also, applications of rigidity in biology and computational science have been reported [9], [10]. Nevertheless, experiments on binary and ternary chalcogenide glasses have shown the existence of two transitions at $\bar{r}_{c 1}$ and $\bar{r}_{c 2}$ instead of the single mean-field transition [11][13]. This suggests that the mean-field constraint counting alone as it has been realized up to now, may be insufficient to describe accurately the underlying phase transitions. Recent results on Raman scattering and modulated differential scanning calorimetry (MDSC) realized on Group IV binary chalcogenides or ternary glasses suggest indeed evidence [14] for the growth of a self-organized (isostatic rigid) intermediate phase between the floppy and the stressed rigid phases, for which evidence is obtained from numerical simulations [15].

However, a certain number of questions remain at this stage. What controls the values $\bar{r}_{c 1}$ and $\bar{r}_{c 2}$, the width $\Delta \bar{r}=\bar{r}_{c 2}-\bar{r}_{c 1}$ ? Recent results show that this width can be particularly sharp [16]. How does isostatic regions and self-organization influence the absolute magnitude of these quantities? What can be done which goes beyond the elegant mean-field approach ? This Letter attempts to adress these basic issues. We report here on the role of medium range order (MRO) in glasses of the form $B_{x} A_{1-x}$ with coordination numbers $r_{A}=2$ and $r_{B}=4$, and $\bar{r}=2+2 x$. Typical glasses are the Group IV chalcogenides such as $G e_{x} S e_{1-x}$ which have been extensively studied in this context. To construct MRO, we have used size-increasing cluster approximations (SICA) to generate sets of clusters on which we have applied constraint counting algorithms. The results show two transitions, one at which the number of floppy modes vanishes. Another transition (a "stress transition") where stress in the structure can not be avoided anymore, is located beyond. In between, this provides evidence for a self-organized network for which the probability of stress-free clusters has been computed. The width $\Delta \bar{r}$ increases with the fraction of MRO elements. Finally, in case of random bonding, a single transition is obtained.

Construction. SICA have been first introduced to elucidate the formation of fullerenes [17], but also the intermediate range order in amorphous semi-conductors [18]. They rely on the statement that the fraction of significant MRO structures converges very rapidly to a limit value when the size of the considered clusters is increasing [19]. The construction is realized in Canonical Ensemble with particular energy levels. One starts from short range order molecules (the basic units at the initial step $l=1$ which will serve as building blocks) and construct all possible structural arrangements of two basic units $(l=2$, see Table I), three basic units $(l=3)$ and so on. This is supposed to be realized at the formation of the network, when $\mathrm{T}$ equals the fictive temperature $T_{f}$ [20]. Here, we have chosen as basic units the $A_{2}$ and the stoichiometric balanced $B A_{2}$ molecules (e.g. $S e_{2}$ and $G e S e_{2}$ ) for a reason which will become clear below. We have checked that the results do not depend on this particular initial choice. These basic units have respective probabilities $1-p$ and 
$p=2 x /(1-x), x$ being the concentration of $\mathrm{B}$ atoms.

The creation of a chain-like $A_{2}-A_{2}$ structure will involve an energy gain of $E_{1}$, isostatic $A_{2}-B A_{2}$ bondings will use an energy gain of $E_{2}$ and the creation of corner-sharing (CS) and edge-sharing (ES) $B A_{4 / 2}$ tetrahedra respectively $E_{3}$ and $E_{4}$. The latter quantity will be used to tune the fraction of ES among the structure. The produced probabilities have different statistical weights which corresponds to the number of equivalent ways a given cluster can be constructed. This quantity can be regarded as the degeneracy of the corresponding energy level. For instance, given the coordination number 4 of the basic unit $B A_{4 / 2}$ and labeled covalent bonds a CS $B_{2} A_{4}$ cluster has the multiplicity $4 \times 4$, whereas for a ES cluster, we count $2 \times\left(\begin{array}{l}4 \\ 2\end{array}\right)\left(\begin{array}{l}4 \\ 2\end{array}\right)=72$ in three dimensions

Due to the initial choice of the basic units, the value of the energy $E_{2}$ will influence the probability of isostatic clusters since this quantity is involved in the probability of the isostatic $B A_{4}$ cluster $\left(n_{c}=3\right.$, see Table I). If we have $e_{2} \gg e_{1}, e_{3}, e_{4}$, the network will be mainly isostatic.

At step $l=2$, we can generate three types of clusters (Table I), $A_{4}, B A_{4}$ and $B_{2} A_{4}$ having two isomers (the CS and ES tetrahedra). Their unrenormalized probabilities are given by: $p_{A_{4}}=4(1-p)^{2} e_{1}, p_{B A_{4}}=16 p(1-p) e_{2}, p_{C S}=16 p^{2} e_{3}$ and $p_{E S}=72 p^{2} e_{4}$ out of which can be extracted the concentration $x^{(l=2)}$ of $B$ atoms. The quantities $e_{i}=\exp \left[-E_{i} / T_{f}\right]$ are the Gibbs weights at $T_{f}$. Next, we compute the number of mechanical constraints (bond-bending and bond-stretching) per atom on each cluster by Maxwell counting. Special care has to be taken in order to avoid the counting of redundant constraints on clusters containing rings, following the procedure described by Thorpe [5]. The probabilites depend on two parameters (i.e. the Gibbs weights $e_{1} / e_{2}$ and $\left.e_{3} / e_{2}\right)$ and eventually $e_{4} / e_{2}$ if one considers the possibility of ES or rings. One of these two weights can be calculated by writing a conservation law for the concentration of $B$ atoms [21]:

$$
x^{(l)}=x
$$

These weights become composition dependent in solving equ. (1.1) which means that either the energies $E_{i}$ or the fictive temperature $T_{f}$ depend on $x$ [20] but here only the $e_{i}(x)$ dependence is relevant for our purpose. With increasing cluster size, it is obvious that the number of potential isomers will increase (Table I), also the different types of rings which have some evidence in chalcogenides [22]. We have realized the construction up to the step $l=4$. At each step, we have determined either $e_{1} / e_{2}$ or $e_{3} / e_{2}$ solving equ. (1.1) and computed the total number of constraints $n_{c}$ per atom on the set of clusters (see Table I). Finally, we have looked for the concentration of $B$ atoms (or the mean coordination number $\bar{r})$ for which the number of floppy modes vanishes.

Results. Random bonding is obtained by setting the above defined Gibbs weights $e_{i}$ to 
one and the cluster probabilities are then only given by their statistical weights.

Solving $f=0$, one obtains a single transition for all steps in the mean coordination number range [2.231,2.275], somewhat lower than the usual mean-field value 2.385 [15]. This comes from the fact that the number of equivalent ways to connect $B A_{4 / 2}$ units together is substantially higher than for the connection of (chain-) $A_{2}$ units. We do not obtain an intermediate phase for random bonding.

Let us turn to self-organization and proceed as follows. Starting from a floppy cluster of size $l$ (almost a chain-like structure made of A atoms), we allow the agglomeration of a new basic unit onto this cluster to generate the cluster of size $l+1$ only if the creation of a stressed rigid region can be avoided on this new cluster (due to the agglomeration of a $B A_{4 / 2}$ basic unit onto another $B A_{4 / 2}$ tetrahedron being part of the $l$-sized cluster). With this rather simple rule, upon increasing $\bar{r}$ we will accumulate isostatic rigid regions on the size increasing clusters because $B A_{4 / 2}$ units are only accepted in $A_{2}-B A_{4 / 2}$ isostatic bondings. Alternatively, we can start from a rigid cluster which exist at higher mean coordination number $(\bar{r} \leq 2.67)$ and follow the same procedure but in opposite way, i.e. we allow only bondings which lead to isostatic rigid regions, excluding systematically the possibility of floppy $A_{2}-A_{2}$ bondings.

Here, the simplest case deals with dendritic clusters, where we have removed all possibilities of ring creation. For $l \rightarrow \infty$, this would permit to recover the results on Random Bond Models [23] for which there are no loops or rings in the thermodynamic limit and to obtain equivalence with Bethe lattice solutions [24]. We obtain a single transition for even $l$ steps at exactly the mean-field value $\bar{r}=2.4$ whereas for the step $l=3$, there is a sharp intermediate phase defined by $f=0$ (again at $\bar{r}=2.4$ ) and the vanishing of floppy regions (i.e. $e_{1} / e_{2}$ is zero) at $\bar{r}=2.382(6)$. The probability of isostatic clusters as a function of the mean coordination number has been computed and shows that the network is entirely stress free at the point where $f=0$ (solid line, fig. 2). If there is a width (for $l=3$ ), then the same probability is less than one and displays a narrow distribution.

Next, we have allowed a certain amount of medium range order (MRO) by setting the quantity $e_{4} / e_{2} \neq 0$. Two transitions are then obtained for every SICA step. A first one at $\bar{r}_{c 1}$ where the number of floppy modes vanishes. A second one at $\bar{r}_{c 2}$ defined by $e_{3} / e_{2}=0$. This means that beyond this point, stressed rigid regions created by the connection of at least two $B A_{4 / 2}$ units can not be avoided anymore, i.e. the Gibbs weight $e_{3} / e_{2}$ becomes non-zero, and composition dependent. We call this point the "stress transition" because its definition is very close to the one given in [15]. We show the $l=2$ result (fig. 2) where $f=0$ at $\bar{r}_{c 1}=2.4$ and $e_{3} \neq 0$ at $\bar{r}>\bar{r}_{c 2}$ for different fractions of ES tetrahedra, defining the intermediate phase $\Delta \bar{r} . \bar{r}_{c 1}$ does not depend on the ES fraction, as well as the fraction of stressed rigid clusters in the structure. To ensure continuous deformation of the network when $\mathrm{B}$ atoms are added and keeping the sum of the probability of floppy, isostatic rigid 
and stressed rigid clusters equal to one, the probability of isostatic rigid clusters connects the isostatic solid line at $\bar{r}_{c 2}$. Stressed rigid rings first appear in the region $\bar{r}_{c 1}<\bar{r}<\bar{r}_{c 2}$ while chain-like stressed clusters (whose probability is proportional to $e_{3}$ ) occur only beyond the stress transition, when $e_{3} \neq 0$. We conclude that when $\bar{r}$ is increased, stressed rigidity nucleates through the network starting from rings. Results remain similar for the even $l=4$ step. It appears from fig. 2 that the width $\Delta \bar{r}=\bar{r}_{c 2}-\bar{r}_{c 1}$ of the intermediate phase increases with the fraction of MRO. We have represented this quantity as a function of the MRO fraction at the rigidity transition in fig. 3 which shows that $\Delta \bar{r}$ is almost an increasing function of the ES fraction as seen from the result at SICA step $l=4$. Here, there is only a small difference between allowing only four-membered rings (ES) (lower dotted line) or rings of all sizes (upper dotted line) in the clusters. Finally, one can see from fig. 2 and the insert of fig. 3 that the probability of isostatic clusters is maximum in the window $\Delta \bar{r}$, and almost equal to 1 for the even SICA steps, providing evidence that the structure is almost stress-free.

Discussion. Chalcogenide glasses represent the ideal systems to check these results. Different types of experimental measurements have given evidence on the two transitions and the nature of the self-organized intermediate phase. Raman scattering has been used 11,13] as a probe to detect elastic thresholds in $S i_{x} S e_{1-x}$ and $G e_{x} S e_{1-x}$ glasses. Specifically, changes in the CS mode chain frequencies have been studied with glass compositions and show a kink (or a jump) at the mean coordination number $\bar{r}_{c 1}=2.4$ and $\bar{r}_{c 2}=2.52$ in Ge and $\bar{r}_{c 2}=2.54$ in Si based systems, suggesting onset of a new rigidity at $\bar{r}_{c 2}$. A clear correlation between these results and the vanishing of the non-reversing heat flow $\Delta H_{n r}$ (the part of the heat flow which is thermal history sensitive) in MDSC measurements has been shown [11,13. Obviously, since this $\Delta H_{n r}$ term provides a measure of how different a glass is from a liquid in a configurational sense, this suggest that in the intermediate phase, glass and liquid structure are closely similar to each other.

The SICA and constraint counting algorithms show that the width $\Delta \bar{r}$ of the intermediate phase increase with the fraction of ES tetrahedra and more generally with MRO composed of small rings (fig. 3). We stress that the width should converge to a lower limit value of $\Delta \bar{r}$ compared to the step $l=2$, therefore one can observe the shift downwards when increasing $l$ from 2 to 4 . This limit value is in principle attained for $l \rightarrow \infty$, or at least for much larger steps than $l=4[18]$. For Si-Se, $\Delta \bar{r}=0.14$ is somewhat larger than forGe-Se $(\Delta \bar{r}=0.12)$ consistently with the fact that the number of ES is higher in the former [13].

In summary, we have shown that size increasing cluster approximations could be used to go beyond the mean-field approach of the rigidity transitions. We have estimated for the different approximation steps the number of mechanical constraints and the number of floppy modes $f$ and shown that two transitions were obtained in this situation. One at which $f$ vanishes and another at which stressed rigid regions appear on the clusters. The 
width $\Delta \bar{r}$ is an increasing function of the MRO fraction. In the window $\Delta \bar{r}$, the rate of isostatic clusters is at its maximum. These new results should motivate developments on the role of local structure and $\mathrm{MRO}$ in the rigidity transition, and applications to Group V chalcogenides such as $A s_{x} S e_{1-x}$ glasses.

The Laboratoire de Physique Théorique des Liquides is Unité Mixte de Recherche n. $7600 \mathrm{du}$ CNRS.

[1] J.L. Lagrange, Mécanique Analytique, Paris (1788)

[2] J.C. Maxwell, Phil. Mag. 27 (1864) 294

[3] J.C. Phillips, J. Non-Cryst. Solids 34 (1979) 153

[4] J.C. Phillips, J. Non-Cryst. Solids 43 (1981) 37

[5] M.F. Thorpe, J. Non-Cryst. Solids 57 (1983) 355; H. He and M.F. Thorpe, Phys. Rev. Lett. 54 (1985) 2107

[6] M. Zhang and P. Boolchand, Science 266 (1994) 1355

[7] P. Boolchand and M.F. Thorpe, Phys. Rev. B50 (1994) 10366

[8] M. Stevens, J. Grothaus, P. Boolchand, J.G. Hernandez, Sol. State Comm. 47 (1983) 199; U. Senapati, A.K. Varshneya, J. Non-Cryst. Solids 185 (1995) 289; T. Wagner and S. Kasap, Phil. Mag. B74 (1996) 667

[9] M.F. Thorpe, M. Lei, A.J. Rader, D.J. Jacobs, L. Kuhn, Proteins 44 (2001) 150

[10] R. Monasson, R. Zecchina, S. Kirkpatrick, B. Selman, L. Troyansky, Nature 400 (1999) 133

[11] X.W. Feng, W.J. Bresser and P. Boolchand, Phys. Rev. Lett. 78 (1997) 4422

[12] D.G. Georgiev, P. Boolchand and M. Micoulaut, Phys. Rev. B62 (2000) 9228

[13] D. Selvenathan, W.J. Bresser, P. Boolchand, Phys. Rev. B61 (2000) 15061

[14] Y. Wang, P. Boolchand, M. Micoulaut, Europhys. Lett. 52 (2000) 633

[15] M.F. Thorpe, D.J. Jacobs, M.V. Chubynsky and J.C. Phillips, J. Non-Cryst. Solids 266-269 (2000) 859

[16] Y. Wang, J. Wells, D.G. Georgiev, P. Boolchand, K. Jackson and M. Micoulaut, Phys. Rev. Lett. 87 (2001) 185503 
[17] R. Kerner, K. Penson and K.H. Bennemann, Europhys. Lett. 19 (1992) 363

[18] M. Micoulaut, R. Kerner and D.M. dos-Santos-Loff, J. Phys. Cond. Matt. 7 (1995) 8035

[19] In fullerenes, one has 12 pentagons and 32 hexagons. The statistics of pentagons and hexagons in clusters of size $n<60$ must therefore converge to the corresponding obvious limit values. In vitreous $\mathrm{B}_{2} \mathrm{O}_{3}$, the fraction of three-membered rings should converge to 0.83 , according to experiment.

[20] F.L. Galeener, D.B. Kerwin, A.J. Miller and J.C. Mikkelsen Jr., Phys. Rev. B47 (1993) 7760

[21] P.J. Bray, S.A. Feller, G.E. Jellison and Y.H. Yun, J. Non-Cryst. Solids 38-39 (1980) 93

[22] J. Griffiths, G. Espinosa, J.C. Phillips, J. Remeika, Phys. Rev. B28 (1983) 4444; A. Pradel, V. Michel-Lledos, M. Ribes and H. Eckert, Chem Mater. 5 (1993) 377

[23] D.J. Jacobs, M.F. Thorpe, Phys. Rev. Lett. 80 (1998) 5451

[24] M.F. Thorpe, D.J. Jacobs, N.V. Chubinsky, Rigidity Theory and Applications Kluwer Academic/Plenum Press (1999) 


\section{List of Figures}

$1 \quad$ Some of the $\mathrm{Ge}_{3} S e_{6}$ and $\mathrm{Ge}_{2} S e_{6} \mathrm{MRO}$ clusters generated by SICA at step $l=3$ with their statistical weight. a) A six-membered ring with $n_{c}=3.67 \mathrm{~b}$ ) An edge-sharing tetrahedra chain typical of vitreous $\mathrm{SiSe}_{2}$ with $n_{c}=3.22 \mathrm{c}$ ) A six-membered ring with chalcogen inclusions and $n_{c}=3.25$. . . . . . . . .

2 Probability of floppy, isostatic rigid and stressed rigid clusters as a function of the mean coordination number for different fractions of ES at $l=2$. The solid lines correspond to the dendritic case where no edge-sharing tetrahedra are allowed. The broken lines correspond to the same quantities for ES fraction at the stress transition of $0.156,0.290$ and 0.818 . For a ES fraction of 0.156 , the filled squares indicate the point $\bar{r}_{c 2}$ at which the stress transition occurs and serves to define the intermediate phase $\Delta \bar{r}$. . . . . . . . . . . . . .

3 Width of the transition $\Delta \bar{r}$ as a function of the fraction of MRO clusters at the rigidity transition for $l=2$ (solid line), $l=3$ (dashed line) and $l=4$ (dotted lines). At step $l=2$, the MRO clusters reduce to the edge-sharing $\mathrm{GeSe}_{4 / 2}$ tetrahedra. For larger steps, different rings sizes $(4,6,8)$ have been taken into account. The lower dotted line correspond to a system at $l=4$ having only ES as MRO element. The insert shows the probability of isostati clusters with mean coordination number $\bar{r}$ for $l=4$ (dotted line) and $l=3$ (dashed line). The shaded region of $l=4$ is defined by the corresponding $\Delta \bar{r}$.

\section{List of Tables}

I Clusters generated at the different SICA steps $l$ with the chemical formula in case of $G e_{x} S e_{1-x}$ glasses, the number of isomers and the number of constraints $n_{c}$ per atom. The number of clusters containing rings is indicated in bracketts. $\mathrm{GeSe}_{4}$ and $\mathrm{Ge}_{2} \mathrm{Se}_{8}$ are isostatic clusters with respective energy levels $E_{2}$ and $2 E_{2} \ldots \ldots \ldots \ldots \ldots \ldots \ldots$ 

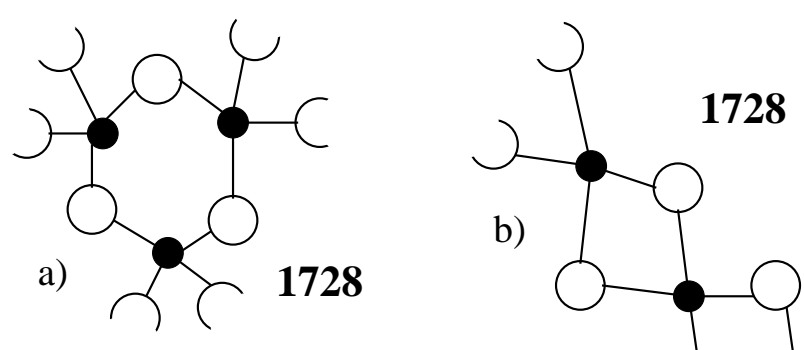

c)

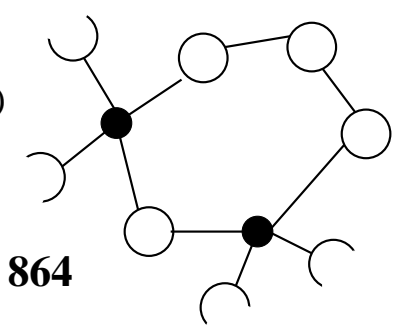

b)

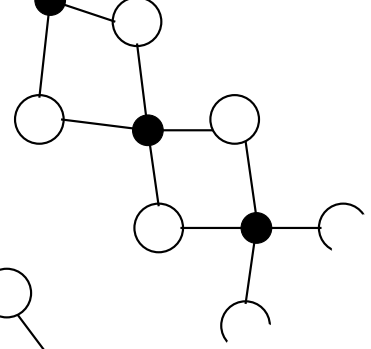

FIG. 1. Some of the $G_{3} \mathrm{Se}_{6}$ and $\mathrm{Ge}_{2} \mathrm{Se}_{6} \mathrm{MRO}$ clusters generated by SICA at step $l=3$ with their statistical weight. a) A six-membered ring with $n_{c}=3.67 \mathrm{~b}$ ) An edge-sharing tetrahedra chain typical of vitreous $\mathrm{SiSe}_{2}$ with $n_{c}=3.22$ c) A six-membered ring with chalcogen inclusions and $n_{c}=3.25$. 


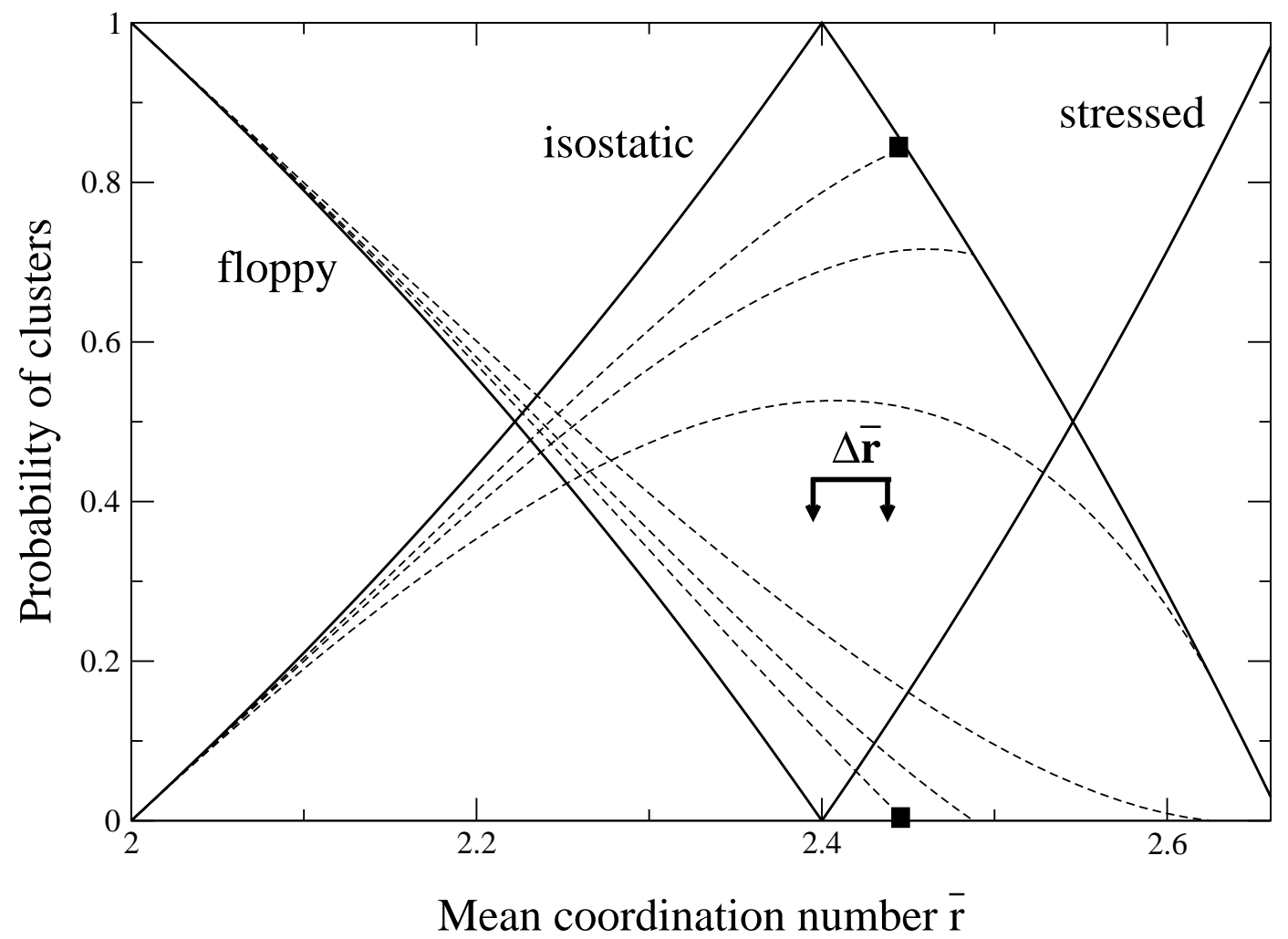

FIG. 2. Probability of floppy, isostatic rigid and stressed rigid clusters as a function of the mean coordination number for different fractions of ES at $l=2$. The solid lines correspond to the dendritic case where no edge-sharing tetrahedra are allowed. The broken lines correspond to the same quantities for ES fraction at the stress transition of 0.156, 0.290 and 0.818. For a ES fraction of 0.156 , the filled squares indicate the point $\bar{r}_{c 2}$ at which the stress transition occurs and serves to define the intermediate phase $\Delta \bar{r}$. 


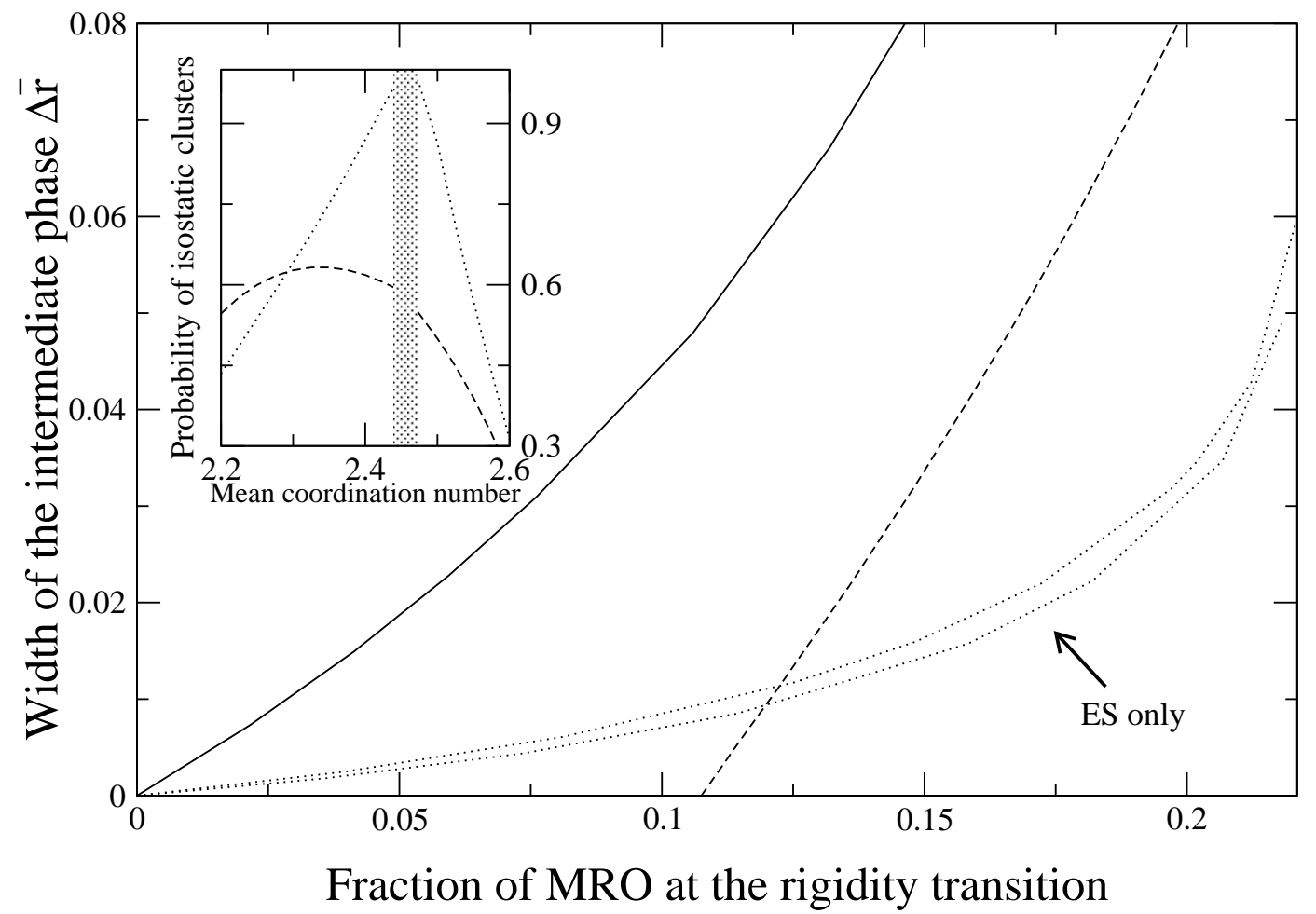

FIG. 3. Width of the transition $\Delta \bar{r}$ as a function of the fraction of MRO clusters at the rigidity transition for $l=2$ (solid line), $l=3$ (dashed line) and $l=4$ (dotted lines). At step $l=2$, the MRO clusters reduce to the edge-sharing $\mathrm{GeSe}_{4 / 2}$ tetrahedra. For larger steps, different rings sizes $(4,6,8)$ have been taken into account. The lower dotted line correspond to a system at $l=4$ having only ES as MRO element. The insert shows the probability of isostatic clusters with mean coordination number $\bar{r}$ for $l=4$ (dotted line) and $l=3$ (dashed line). The shaded region of $l=4$ is defined by the corresponding $\Delta \bar{r}$. 


\begin{tabular}{cccc}
\hline \hline Size $l$ & cluster & Number of isomers & $n_{c}$ \\
\hline 1 & $\mathrm{Se}_{2}$ & 1 & 2 \\
& $\mathrm{GeSe}_{2}$ & 1 & 3.67 \\
\hline 2 & $\mathrm{Se}_{4}$ & 1 & 2 \\
& $\mathrm{GeSe}_{4}$ & 1 & 3 \\
& $\mathrm{Ge}_{2} \mathrm{Se}_{4}$ & $2(1)$ & 3.67 \\
\hline 3 & $\mathrm{Se}_{6}$ & 1 & 2 \\
& $\mathrm{GeSe}_{6}$ & 2 & 2.71 \\
& $\mathrm{Ge}_{2} \mathrm{Se}_{6}$ & $4(2)$ & 3.25 \\
& $\mathrm{Ge}_{3} \mathrm{Se}_{6}$ & $4(3)$ & 3.67 \\
\hline 4 & $\mathrm{Se}_{8}$ & 1 & 2 \\
& $\mathrm{GeSe}_{8}$ & 3 & 2.56 \\
& $\mathrm{Ge}_{2} \mathrm{Se}_{8}$ & $11(6)$ & 3 \\
& $\mathrm{Ge}_{3} \mathrm{Se}_{8}$ & $12(9)$ & 3.36 \\
& $\mathrm{Ge}_{4} \mathrm{Se}_{8}$ & $10(9)$ & 3.67 \\
\hline \hline
\end{tabular}

TABLE I. Clusters generated at the different SICA steps $l$ with the chemical formula in case of $G e_{x} S e_{1-x}$ glasses, the number of isomers and the number of constraints $n_{c}$ per atom. The number of clusters containing rings is indicated in bracketts. $\mathrm{GeSe}_{4}$ and $\mathrm{Ge}_{2} \mathrm{Se}_{8}$ are isostatic clusters with respective energy levels $E_{2}$ and $2 E_{2}$. 